\title{
Blood Flow Restriction Therapy and Its Use for Rehabilitation and Return to Sport: Physiology, Application, and Guidelines for Implementation
}

\author{
Daniel J. Cognetti, M.D., Andrew J. Sheean, M.D., and Johnny G. Owens, M.P.T.
}

\begin{abstract}
Blood flow restriction (BFR) is an expanding rehabilitation modality that uses a tourniquet to reduce arterial inflow and occlude venous outflow in the setting of resistance training or exercise. Initially, this technique was seen as a way to stimulate muscular development, but improved understanding of its physiologic benefits and mechanism of action has allowed for innovative clinical applications. BFR represents a way to decrease stress placed on the joints without compromising improvements in strength, whereas for postoperative, injured, or load-compromised individuals BFR represents a way to accelerate recovery and prevent atrophy. There is also growing evidence to suggest that it augments cardiovascular fitness and attenuates pain. The purpose of this review is to highlight the physiology and evidence behind the various applications of BFR, with a focus on postoperative rehabilitation. While much remains to be learned, it is clear that blood flow restriction therapy stimulates muscle hypertrophy via a synergistic response to metabolic stress and mechanical tension, with supplemental benefits on cardiovascular fitness and pain. New forms of BFR and expanding applications in postoperative patients and athletes hold promise for expedited recovery. Continued adherence to rehabilitation guidelines and exploration of BFRs physiology and various applications will help optimize its effect and prescription. Level of Evidence: V, expert opinion.
\end{abstract}

B lood flow restriction (BFR) therapy, a controlled form of vascular occlusion combined with resistance training or exercise, has seen tremendous growth in recent years. Initially, this modality of rehabilitation gained notoriety for its use in wounded servicemembers with volumetric muscle loss and limb-salvage scenarios, but it has expanded across a number of applications, including regular strength training, postoperative rehabilitation, and atrophy prevention. Other novel forms being applied clinically also include BFR

From the Department of Orthopedics and Rehabilitation, San Antonio Military Medical Center (D.J.C., A.J.S.); and Owens Recovery Science (J.G.O.), San Antonio, Texas, U.S.A.

The authors report the following potential conflicts of interest or sources of funding: A.J.S. reports grants from Embody and personal fees from Arthroscopy, outside the submitted work. J.G.O. reports personal fees from Major Extremity Trauma Readiness Consortium and Delfi Medical Innovations, outside the submitted work. Full ICMJE author disclosure forms are available for this article online, as supplementary material.

Received August 2, 2021; accepted September 22, 2021.

Address correspondence to Daniel J. Cognetti, M.D., 3551 Roger Brooke Dr., San Antonio, TX 78234.E-mail: cognettidj@gmail.com

Published by Elsevier Inc. on behalf of the Arthroscopy Association of North America. This is an open access article under the CC BY-NC-ND license (http://creativecommons.org/licenses/by-nc-nd/4.0/).

2666-061X/211126

https://doi.org/10.1016/j.asmr.2021.09.025 with aerobic exercise, passive application (i.e., BFR in the absence of exercise), and neuromuscular stimulation. As awareness of BFR has grown so too has the knowledge behind its physiologic mechanism of action. This has uncovered multiple benefits beyond that of muscular growth, including improvement in muscular endurance, cardiovascular fitness, pain, and bone density. ${ }^{1-3}$ The purpose of this review is to highlight the physiology and evidence behind the various applications of BFR, with a focus on postoperative rehabilitation. Consideration also will be given to guidelines for safe implementation.

\section{Physiology/Mechanism of Action}

Before exploring the applications and practical guidelines for implementing BFR, it is important to understand its mechanism of action. A number of mechanisms have been theorized, but currently it is believed that metabolic stress from vascular occlusion and mechanical tension from resistance training or exercise lead to synergistic increases in muscle hypertrophy and strength. ${ }^{4-9}$ At a cellular level, metabolites, hormonal differences, cell-to-cell signaling, cellular swelling, and intracellular signaling pathways have all been implicated. ${ }^{5,6,10-13}$ Metabolites, which accumulate during exercise and are known mediators of muscular 


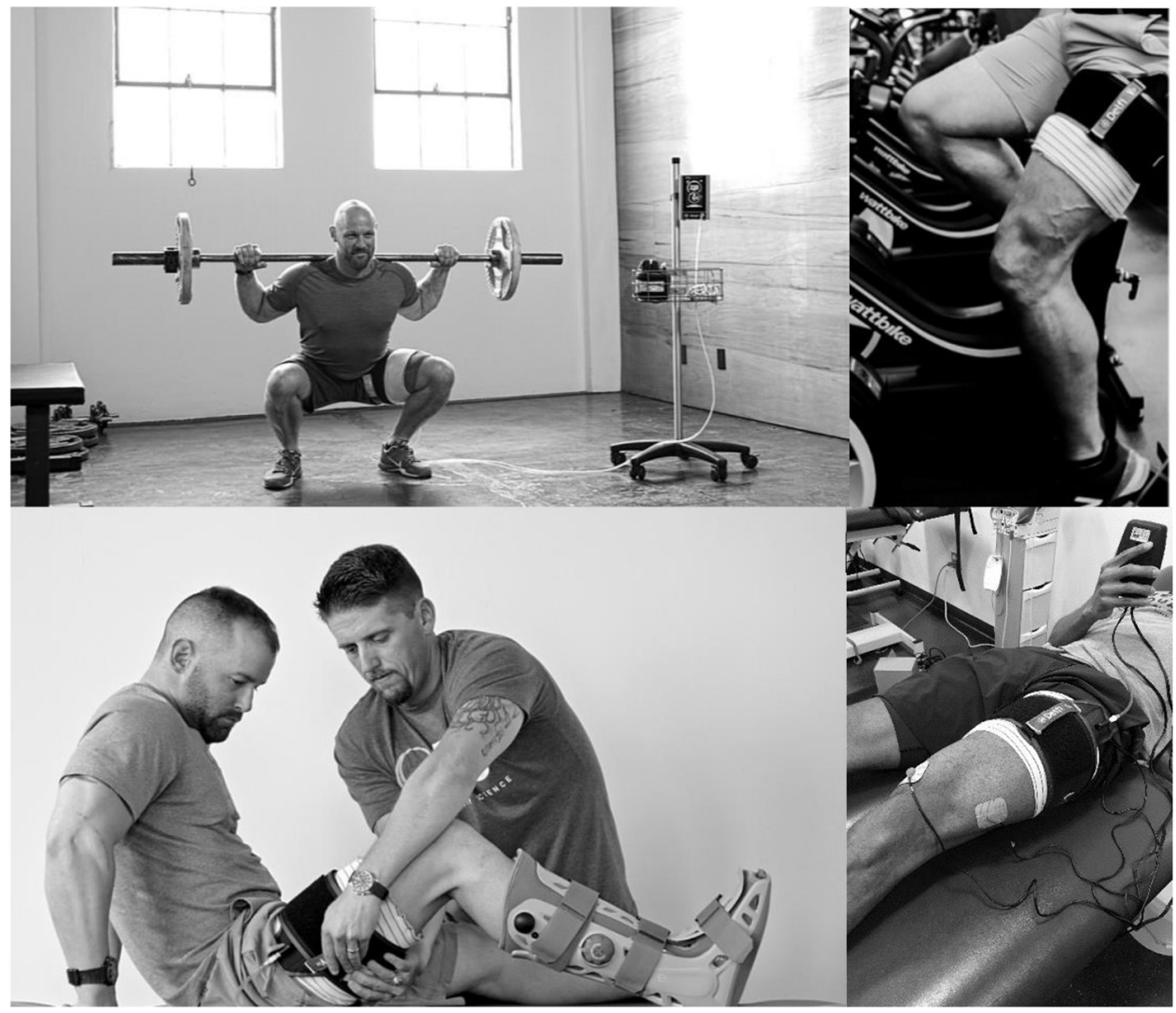

Fig 1. Blood flow restriction with resistance training, aerobic exercise, passive application, and neuromuscular stimulation. hypertrophy, are amplified by BFR's relative ischemic and hypoxic conditions. They are believed to induce earlier, peripherally mediated fatigue, resulting in greater motor unit recruitment, as suggested by the fact that BFR under low loads has similar recruitment to that of high load resistance training. ${ }^{7,14,15}$ In addition, type II fast-twitch muscle fibers, which are normally only preferentially recruited at greater intensity, are activated at lower loads under BFR conditions, providing rationale for the increased muscle hypertrophy in low-load BFR in comparison with similar lowload exercise alone. ${ }^{16-18}$ However, greater motor unit recruitment is not limited to muscles distal to the area of occlusion. In both upper- and lower-extremity BFR, more proximal muscle groups (gluteus maximus, shoulder [deltoid/rotator cuff], and pectoralis major) have been shown to have greater levels of recruitment as compared with controls. ${ }^{19-23}$ This is hypothesized to occur in response to the early fatigue of synchronous muscle groups distal to the occlusion site and has important implications in BFR's use after procedures or injuries where a tourniquet cannot be applied proximally.

The supraphysiologic benefits of exercise with BFR also may be partially explained by the proliferation of satellite cells, multipotent cells within muscle connective tissue responsible for muscle growth and regeneration. Although satellite cells initially were thought to only be activated in the setting of highresistance training, their proliferation is increased even under low loads with BFR, with associated increases in muscle protein synthesis, myonuclei content, myofiber size, and muscle strength. ${ }^{16,24,25}$

The physiologic adaptations noted during and after BFR also have been found to be mediated by several notable cell signaling pathways. Stimulation of protein translation via the mechanistic target of rapamycin pathway, which is important in muscle protein synthesis and hypertrophy, ${ }^{26-28}$ appears to play a fundamental role in the effects of BFR, ${ }^{11,12}$ whereas myostatin, a negative regulator of muscle growth and promoter of muscle fibrosis, has been shown to be downregulated after BFR. ${ }^{13,29,30}$ Although the exact contribution of each mechanistic pathway may not yet be completely understood, the available evidence offers important insight which will help guide further research to optimize rehabilitation efforts.

\section{Applications}

In its earliest applications, BFR was used to stimulate muscular growth, but as word of its potential has disseminated so too have the applications and indications for its use. Current forms include BFR with low-load resistance training, aerobic exercise, passive 
BFR, and neuromuscular stimulation (Fig 1). These various modalities have been studied across a variety of demographic subgroups including athletes and nonathletes, healthy and injured, preoperative and postoperative, young and old.

By far the most common form of BFR is with resistance exercise (BRF-RE), with multiple systematic reviews and meta-analyses demonstrating its benefits. ${ }^{31-34}$ From these studies it is clear that low-load BFR-RE is superior in multiple respects, including building muscle strength and size, to that of isolated low load resistance training and comparable to that of high load resistance training alone. However, although lowload BFR-RE may not necessarily be superior to that of high-load resistance training in terms of muscle strength, the ability to train at a greater frequency with less mechanical stress on the joints allows for broad application, in postoperative, injured, and cardiac rehabilitation patients, in season athletes and elderly individuals.

In the interest of providing concise, practical recommendations for producing muscle hypertrophy with BFR-RE, $20 \%$ to $40 \%$ of 1 repetition maximums are recommended in concert with BFR cuff pressures set between $40 \%$ and $80 \%$ of limb occlusion pressure (LOP). ${ }^{35}$ Four sets of repetitions $(30,15,15,15)$ has been most commonly used in practice and produces the beneficial adaptions noted in BFR. Generally, rest between sets of 30 to 60 seconds is recommended, with concern that longer periods and intermittent BFR (relieving the cuff pressure between sets) may limit the stress for adaption. ${ }^{35}$ Two to three BFR sessions per week are recommended, in line with recommendations for skeletal muscle hypertrophy in standard resistance training of 2 to 4 times per week. A more aggressive regimen, including twice-daily BFR training, may be a reasonable option to accelerate recovery in the early rehabilitation period following an injury or surgery. ${ }^{35}$

A hallmark of returning patients to activity or sports is improvement and maintenance of their aerobic fitness. The postoperative period can severely diminish aerobic capacity and in hospitalized or elderly individuals this component of physical health can be the crux of functional recovery. The study of BFR with aerobic exercise (BFR-AE) has been more limited compared with resistance training, but markers of aerobic fitness, including absolute and relative oxygen consumption $\left(\mathrm{VO}_{2}\right)$, along with exercise time to exhaustion, have all been shown to increase more compared to control training groups. ${ }^{1,36}$ In addition, low-intensity exercise in combination with BFR has shown similar substantial improvements in $\mathrm{VO}_{2}$ and strength even in already highly trained athletes. ${ }^{37-39}$ While it is often felt that a threshold of $50 \% \mathrm{VO}_{2}$ should be reached to improve aerobic capacity, a clinical protocol for BFR-AE of $40 \%$ $\mathrm{VO}_{2}$ maximum (or walking at approximately 2-4 miles per hour) for as little as 15 to 20 minutes may be able to achieve similar effects over 2 to 6 weeks. ${ }^{36,39,40}$ These effects are believed to be mediated by a decrease in venous return, which is compensated for by increases in heart rate, resulting in adjustments to the effective window for aerobic adaptation. While not necessarily the main focus of BFR-AE, improvements in strength, muscle size, glycogen composition, capillary fiber density, and functional measures, such as timed-up and-go and chair sit-to-stand, also have been noted across various studies. ${ }^{36,39-42}$

Although the benefits of BFR and exercise are felt to be most beneficial in tandem, BFR's positive effect has been shown to be present in the absence of exercise as well. For individuals in the postoperative period, immobilized, casted or hospitalized, BFR alone represents a potential solution to prevent disuse atrophy. A systematic review of 3 studies in non-weight-bearing or immobilized individuals ( 1 following anterior cruciate ligament reconstruction [ACLR] and 2 for short-leg cast immobilization and non-weight-bearing in healthy individuals) showed BFR attenuated reductions in knee torque and prevented disuse atrophy. However, the conclusions from the review itself were limited due to high risk of bias of each study. ${ }^{43}$ One other study looking at intensive care patients in comas undergoing BFR with passive mobilization, using the contralateral limb as a control, showed BFR was an effective way to reduce muscle wasting from the thigh. However, across the studies looking at BFR alone and with passive motion, large ranges of pressure have been applied without relative reference numbers such as LOP, limiting standardization and generalizability of the findings. Nevertheless, it appears that high pressures (70\%-100\% LOP) provide a stronger stimulus to prevent atrophy, while the most common protocol in these scenarios is 5 minutes of BFR, followed by 3 minutes of reperfusion, for 3 to 4 cycles, once or twice a day for 1 to 8 weeks. ${ }^{35}$

Another budding area of interest includes the use of BFR with neuromuscular stimulation. Neuromuscular stimulation often is used in the early postoperative period and as maintenance/preventative therapy in training rooms for collegiate and professional athletes. Early evidence suggests the effect of this therapy, which provides unloaded, isometric contractions, may be enhanced by BFR. ${ }^{33,44}$ With further study, this could prove useful as an adjunct in the early recovery period following knee surgery where quadriceps control is often a limiting factor in rehabilitation progression.

\section{Postoperative Rehabilitation}

The rapid onset and significant burden of atrophy following surgery and/or immobilization is well documented, as are the detrimental effects of atrophy on recovery, overall function, and reinjury. ${ }^{45-47}$ With 
immobilization alone, healthy, nonsurgical patients had a $7 \%$ decrease in quadriceps muscle at 7 days, with atrophy after knee surgery being comparatively greater, up to $33 \%$ at 3 weeks. ${ }^{48-50}$ The benefits of low load (or no load) BFR are perfectly suited for this population given improved skeletal muscle maintenance, less associated muscle damage postexercise, ${ }^{51}$ hypoalgesia, ${ }^{52}$ and decreased mechanical stress on the joints.

BFR is being investigated after both upper- and lower-extremity surgery, but most notably significant interest has been paid to ACLR. Multiple randomized controlled trials with varying postoperative protocols have observed somewhat differing results, but it appears that low-load BFR applied within the first few weeks after surgery is effective at improving muscular size and strength over standard rehabilitation. ${ }^{53}$ More recent studies have also shown improvements in kneespecific patient-reported outcomes, as well as reduced knee pain and effusions, compared with standard rehabilitation. ${ }^{54,55}$ Although the majority of research has focused on mitigating muscle loss acutely, BFR also has shown benefit in more chronic postoperative deficits. In individuals with severe chronic thigh weakness after knee surgery the majority made significant increases in strength after just 9 BFR sessions. ${ }^{56}$ In addition, one study found that patients on average 5 years out from ACLR with mild persistent quadriceps deficits were able to benefit from BFR. ${ }^{57}$ A scoping review of the literature pointed out that while most of these studies contain measures of muscle volume and/or strength, these measures are of limited value in assessing benefits to functionality. ${ }^{58}$ One other notable limitation of the current literature is that there are few studies of BFR in ACLR that use autografts derived from the extensor mechanism, whether quadriceps tendon or patellar tendon. This is particularly relevant as the quadriceps muscle is likely more prone to atrophy in these scenarios, which may potentiate the benefit of BFR.

While prescription of BFR after ACLR has been shown to be effective, BFR may also be warranted in instances of prolonged non-weight-bearing/ immobilization (meniscal repair, meniscal transplant, articular cartilage procedures, osteotomy), multiligamentous reconstructions or in those individuals with prolonged and refractory atrophy. Evidence of BFR's benefit for muscle groups proximal to the site of occlusion also has expanded its potential use for shoulder and hip procedures, but before advocating for routine prescription from arthroscopy to arthroplasty, further evidence of clinical benefit is needed. ${ }^{19-21,59}$

\section{Safety Considerations}

While remarkable to see the influence of scientific evidence on athletic performance, some caution is warranted given the risks associated with inappropriate
BFR usage. This is especially true, given uncertainty regarding its effectiveness in scenarios in which pressure is applied via less-precise methods than pneumatic tourniquets with calibrated pressures. Temporary paresthesias, bruising, and delayed-onset muscle soreness all may result after routine BFR use, but serious adverse events, including rhabdomyolysis, prolonged pain, and syncopal events, can result from inappropriate utilization, over exertion, or in individuals who are not healthy enough for moderate or intense physical activity. ${ }^{60}$ Lastly, while blood clots were often cited as a risk in early studies and discussion of BFR, there is no evidence to support an increased risk of thromboembolic events. ${ }^{61}$ In fact, the opposite may even be true, with BFR offering a protective effect against such events, given its stimulation of the fibrinolytic system. These points notwithstanding, implementation of BFR, within consensus guidelines, does not appear to increase the risk of adverse events any more than standard exercise modalities.

\section{Conclusions}

BFR therapy stimulates muscle hypertrophy via a synergistic response to metabolic stress and mechanical tension, with supplemental benefits on cardiovascular fitness and pain. New forms of BFR and expanding applications in postoperative patients and athletes hold promise for expedited recovery. Continued adherence to rehabilitation guidelines and exploration of BFRs physiology and various applications will help optimize its effect and prescription.

\section{References}

1. Formiga MF, Fay R, Hutchinson S, et al. Effect of aerobic exercise training with and without blood flow restriction on aerobic capacity in healthy young adults: A systematic review with meta-analysis. Int J Sports Phys Ther 2020;15: 175-187.

2. Hughes L, Patterson SD. The effect of blood flow restriction exercise on exercise-induced hypoalgesia and endogenous opioid and endocannabinoid mechanisms of pain modulation. J Appl Physiol 2020;128:914-924.

3. Lambert B, Hedt CA, Jack RA, et al. Blood flow restriction therapy preserves whole limb bone and muscle following ACL reconstruction. Orthop J Sports Med 2019;7: 232596711950019 (3 suppl 2).

4. Goto K, Ishii N, Kizuka T, Takamatsu K. The impact of metabolic stress on hormonal responses and muscular adaptations. Med Sci Sports Exerc 2005;37:955-963.

5. Suga T, Okita K, Morita N, et al. Intramuscular metabolism during low-intensity resistance exercise with blood flow restriction. J Appl Physiol 2009;106:1119-1124.

6. Takarada Y, Nakamura Y, Aruga S, Onda T, Miyazaki S, Ishii N. Rapid increase in plasma growth hormone after low-intensity resistance exercise with vascular occlusion. J Appl Physiol2000;88:61-65. 
7. Schoenfeld BJ. The mechanisms of muscle hypertrophy and their application to resistance training. J Strength Cond Res 2010;24:2857-2872.

8. Zou K, Meador BM, Johnson B, et al. The $\alpha_{7} \beta_{1}$-integrin increases muscle hypertrophy following multiple bouts of eccentric exercise. J Appl Physiol 2011;111:1134-1141.

9. Tatsumi R, Liu X, Pulido A, et al. Satellite cell activation in stretched skeletal muscle and the role of nitric oxide and hepatocyte growth factor. Am J Physiol Cell Physiol 2006;290:C1487-C1494.

10. Reeves GV, Kraemer RR, Hollander DB, et al. Comparison of hormone responses following light resistance exercise with partial vascular occlusion and moderately difficult resistance exercise without occlusion. J Appl Physiol 2006;101:1616-1622.

11. Fry CS, Glynn EL, Drummond MJ, et al. Blood flow restriction exercise stimulates mTORCl signaling and muscle protein synthesis in older men. J Appl Physiol 2010;108:1199-1209.

12. Fujita S, Abe T, Drummond MJ, et al. Blood flow restriction during low-intensity resistance exercise increases S6K1 phosphorylation and muscle protein synthesis. J Appl Physiol 2007;103:903-910.

13. Laurentino GC, Ugrinowitsch C, Roschel H, et al. Strength training with blood flow restriction diminishes myostatin gene expression. Med Sci Sports Exerc 2012;44:406-412.

14. Hwang PS, Willoughby DS. Mechanisms behind blood flow-restricted training and its effect toward muscle growth. J Strength Cond Res 2019;33:S167-S179.

15. Jessee MB, Mattocks KT, Buckner SL, et al. Mechanisms of blood flow restriction: The new testament. Tech Orthop 2018:33:72-79.

16. Wernbom M, Apro W, Paulsen G, Nilsen TS, Blomstrand E, Raastad T. Acute low-load resistance exercise with and without blood flow restriction increased protein signalling and number of satellite cells in human skeletal muscle. Eur J Appl Physiol 2013;1 13:2953-2965.

17. Takarada Y, Sato Y, Ishii N. Effects of resistance exercise combined with vascular occlusion on muscle function in athletes. Eur J Appl Physiol 2002;86:308-314.

18. Yasuda T, Brechue WF, Fujita T, Shirakawa J, Sato Y, Abe T. Muscle activation during low-intensity muscle contractions with restricted blood flow. J Sports Sci 2009;27:479-489.

19. Bowman EN, Elshaar R, Milligan H, et al. Upper-extremity blood flow restriction: The proximal, distal, and contralateral effects-a randomized controlled trial. J Shoulder Elbow Surg 2020;29:1267-1274.

20. Dankel SJ, Jessee MB, Abe T, Loenneke JP. The effects of blood flow restriction on upper-body musculature located distal and proximal to applied pressure. Sports Med 2016;46:23-33.

21. Lambert B, Hedt C, Daum J, et al. Blood flow restriction training for the shoulder: A case for proximal benefit. Am J Sports Med 2021;49:2716-2728.

22. Yasuda T, Fujita S, Ogasawara R, Sato Y, Abe T. Effects of low-intensity bench press training with restricted arm muscle blood flow on chest muscle hypertrophy: A pilot study. Clin Physiol Funct Imaging 2010;30:338-343.

23. Yasuda T, Fujita T, Miyagi Y, et al. Electromyographic responses of arm and chest muscle during bench press exercise with and without KAATSU. Int $J$ KAATSU Training Res 2006;2:15-18.

24. Nielsen JL, Aagaard P, Bech RD, et al. Proliferation of myogenic stem cells in human skeletal muscle in response to low-load resistance training with blood flow restriction: Elevation of myogenic stem cells with blood flow restricted exercise. J Appl Physiol 2012;590:4351-4361.

25. Bjørnsen T, Wernbom M, Løvstad A, et al. Delayed myonuclear addition, myofiber hypertrophy, and increases in strength with high-frequency low-load blood flow restricted training to volitional failure. J Appl Physiol 2019;126:578-592.

26. Baar K, Esser K. Phosphorylation of $\mathrm{p} 70^{\mathrm{S} 6 \mathrm{k}}$ correlates with increased skeletal muscle mass following resistance exercise. Am J Physiol Cell Physiol 1999;276:C120-C127.

27. Bodine SC, Stitt TN, Gonzalez $M$, et al. Akt/mTOR pathway is a crucial regulator of skeletal muscle hypertrophy and can prevent muscle atrophy in vivo. Nat Cell Biol 2001;3:1014-1019.

28. Reynolds TH, Bodine SC, Lawrence JC. Control of Ser2448 phosphorylation in the mammalian target of rapamycin by insulin and skeletal muscle load. J Biol Chem 2002;277:17657-17662.

29. Kawada S, Ishii N. Skeletal muscle hypertrophy after chronic restriction of venous blood flow in rats. Med Sci Sports Exerc 2005;37:1144-1150.

30. Drummond MJ, Fujita S, Takashi A, Dreyer HC, Volpi E, Rasmussen BB. Human muscle gene expression following resistance exercise and blood flow restriction. Med Sci Sports Exerc 2008;40:691-698.

31. Hughes L, Paton B, Rosenblatt B, Gissane C, Patterson SD. Blood flow restriction training in clinical musculoskeletal rehabilitation: A systematic review and meta-analysis. $\mathrm{Br}$ J Sports Med 2017;51:1003-1011.

32. Centner C, Wiegel P, Gollhofer A, König D. Effects of blood flow restriction training on muscular strength and hypertrophy in older individuals: A systematic review and meta-analysis. Sports Med 2019;49:95-108.

33. Slysz J, Stultz J, Burr JF. The efficacy of blood flow restricted exercise: A systematic review \& meta-analysis. J Sci Med Sport 2016;19:669-675.

34. Lixandrão ME, Ugrinowitsch C, Berton R, et al. Magnitude of muscle strength and mass adaptations between high-load resistance training versus low-load resistance training associated with blood-flow restriction: A systematic review and meta-analysis. Sports Med 2018;48: 361-378.

35. Patterson SD, Hughes L, Warmington S, et al. Blood flow restriction exercise: considerations of methodology, application, and safety. Front Physiol 2019;10:533.

36. Abe T, Fujita S, Nakajima T, et al. Effects of low-intensity cycle training with restricted leg blood flow on thigh muscle volume and VO2MAX in young men. J Sports Sci Med 2010;9:452-458.

37. Held S, Behringer M, Donath L. Low intensity rowing with blood flow restriction over 5 weeks increases VO2max in elite rowers: A randomized controlled trial. J Sci Med Sport 2020;23:304-308.

38. Ursprung W, Smith JD. The effects of blood flow restriction training on VO2Max and 1.5 mile run performance. Int J Exerc Sci Conf Proc 2017;2:108. 
39. Park S, Kim JK, Choi HM, Kim HG, Beekley MD, Nho H. Increase in maximal oxygen uptake following 2-week walk training with blood flow occlusion in athletes. Eur J Appl Physiol 2010;109:591-600.

40. Abe T, Kearns CF, Sato Y. Muscle size and strength are increased following walk training with restricted venous blood flow from the leg muscle, Kaatsu-walk training. J Appl Physiol 2006;100:1460-1466.

41. Sundberg CJ, Eiken O, Nygren A, Kaijser L. Effects of ischaemic training on local aerobic muscle performance in man. Acta Physiol Scand 1993;148:13-19.

42. de Oliveira MFM, Caputo F, Corvino RB, Denadai BS. Short-term low-intensity blood flow restricted interval training improves both aerobic fitness and muscle strength: Functional gains and short-term training. Scand J Med Sci Sports 2016;26:1017-1025.

43. Cerqueira MS, Do Nascimento JDS, Maciel DG, Barboza JAM, De Brito Vieira WH. Effects of blood flow restriction without additional exercise on strength reductions and muscular atrophy following immobilization: A systematic review. J Sport Health Sci 2020;9:152-159.

44. Natsume T, Ozaki H, Saito AI, Abe T, Naito H. Effects of electrostimulation with blood flow restriction on muscle size and strength. Med Sci Sports Exerc 2015;47:2621-2627.

45. Lepley LK. Deficits in quadriceps strength and patientoriented outcomes at return to activity after ACL reconstruction: A review of the current literature. Sports Health 2015;7:231-238.

46. Shaw T, Williams MT, Chipchase LS. Do early quadriceps exercises affect the outcome of ACL reconstruction? A randomised controlled trial. Aust J Physiother 2005;51:9-17.

47. Chmielewski TL, Wilk KE, Snyder-Mackler L. Changes in weight-bearing following injury or surgical reconstruction of the ACL: Relationship to quadriceps strength and function. Gait Posture 2002;16:87-95.

48. Iversen E, Røstad V, Larmo A. Intermittent blood flow restriction does not reduce atrophy following anterior cruciate ligament reconstruction. J Sport Health Sci 2016;5: $115-118$.

49. Gerber JP, Marcus RL, Dibble LE, LaStayo PC. The use of eccentrically biased resistance exercise to mitigate muscle impairments following anterior cruciate ligament reconstruction: A short review. Sports Health 2009;1:31-38.

50. Kilroe SP, Fulford J, Jackman SR, Van Loon LJC, Wall BT. Temporal muscle-specific disuse atrophy during one week of leg immobilization. Med Sci Sports Exerc 2020;52: 944-954.
51. Loenneke JP, Thiebaud RS, Abe T. Does blood flow restriction result in skeletal muscle damage? A critical review of available evidence: Blood flow restriction. Scand J Med Sci Sports 2014;24:e415-422.

52. Song JS, Spitz RW, Yamada Y, et al. Exercise-induced hypoalgesia and pain reduction following blood flow restriction: A brief review. Physical Ther Sport 2021;50: 89-96.

53. Lu Y, Patel BH, Kym C, et al. Perioperative blood flow restriction rehabilitation in patients undergoing ACL reconstruction: A systematic review. Orthop J Sports Med 2020;8:232596712090682.

54. Hughes L, Patterson SD, Haddad F, et al. Examination of the comfort and pain experienced with blood flow restriction training during post-surgery rehabilitation of anterior cruciate ligament reconstruction patients: A UK National Health Service trial. Phys Ther Sport 2019;39: 90-98.

55. Hughes L, Rosenblatt B, Haddad F, et al. Comparing the effectiveness of blood flow restriction and traditional heavy load resistance training in the post-surgery rehabilitation of anterior cruciate ligament reconstruction patients: A UK National Health Service randomised controlled trial. Sports Med 2019;49:1787-1805.

56. Noyes FR, Barber-Westin SD, Sipes L. Blood flow restriction training can improve peak torque strength in chronic atrophic postoperative quadriceps and hamstrings muscles. Arthroscopy 2021;37:2860-2869.

57. Kilgas MA, Lytle LLM, Drum SN, Elmer SJ. Exercise with blood flow restriction to improve quadriceps function long after ACL reconstruction. Int J Sports Med 2019;40: 650-656.

58. Caetano D, Oliveira C, Correia C, Barbosa P, Montes A, Carvalho P. Rehabilitation outcomes and parameters of blood flow restriction training in ACL injury: A scoping review. Phys Ther Sport 2021;49:129-137.

59. Brumitt J, Hutchison MK, Kang D, et al. Blood flow restriction training for the rotator cuff: A randomized controlled trial. Int J Sports Physiol Perform 2020;15: 1175-1180.

60. Tabata S, Suzuki Y, Azuma K, Matsumoto H. Rhabdomyolysis after performing blood flow restriction training: A case report. J Strength Cond Res 2016;30:2064-2068.

61. Nascimento D da C, Petriz B, da Cunha Oliveira S, et al. Effects of blood flow restriction exercise on hemostasis: A systematic review of randomized and non-randomized trials. Int J Gen Med 2019;12:91-100. 